\title{
Magnetic impurities in gapless Fermi systems: perturbation theory
}

\author{
Matthew T Glossop and David E Logan \\ Oxford University, Physical and Theoretical Chemistry Laboratory, South Parks \\ Road, Oxford OX1 3QZ, UK
}

\begin{abstract}
We consider a symmetric Anderson impurity model with a soft-gap hybridization vanishing at the Fermi level, $\Delta_{\mathrm{I}} \propto|\omega|^{r}$ with $r>0$. Three facets of the problem are examined. First the non-interacting limit, which despite its simplicity contains much physics relevant to the $U>0$ case: it exhibits both strong coupling (SC) states (for $r<1$ ) and local moment states (for $r>1$ ), with characteristic signatures in both spectral properties and thermodynamic functions. Second, we establish general conditions upon the interaction self-energy for the occurence of a SC state for $U>0$. This leads to a pinning theorem, whereby the modified spectral function $A(\omega)=|\omega|^{r} D(\omega)$ is pinned at the Fermi level $\omega=0$ for any $U$ where a SC state obtains; it generalizes to arbitrary $r$ the pinning condition upon $D(\omega=0)$ familiar in the normal $r=0$ Anderson model. Finally, we consider explicitly spectral functions at the simplest level: second order perturbation theory in $U$, which we conclude is applicable for $r<\frac{1}{2}$ and $r>1$ but not for $\frac{1}{2}<r<1$. Characteristic spectral features observed in numerical renormalization group calculations are thereby recovered, for both SC and LM phases; and for the SC state the modified spectral functions are found to contain a generalized Abrikosov-Suhl resonance exhibiting a characteristic low-energy Kondo scale with increasing interaction strength.
\end{abstract}




\section{Introduction}

The Kondo effect, whereby an impurity spin is quenched by coupling to the low-energy excitations of a non-interacting metallic host, has long occupied a central role in the study of magnetic impurities (see e.g. [1]). The effect is of course normally regarded as being dependent upon a metallic host, with low-energy and hence low-temperature impurity properties controlled by the non-vanishing host density of states at the Fermi level, $\omega=0$, and essentially independent of the details of host band structure.

But what if the host exhibits semi-metallic character, with a spectrum whose lowenergy behaviour exhibits a soft-gap at the Fermi level, $\rho_{\text {host }} \propto|\omega|^{r}$ with $r>0$ ? There are quite a number of experimental candidates for such behaviour, ranging from semiconductors whose valence and conduction bands touch at the Fermi level [2], through heavy Fermion superconductors [3], to various two-dimensional systems including graphite sheets [4] and quasi-one-dimensional metals described by a Luttinger model [5].

The question above was first posed by Withoff and Fradkin [6], who studied the soft-gap Kondo model using a combination of 'poor man's' scaling and a large- $N$ meanfield theory (with $N$ the impurity degeneracy). Since then, there has been much study of both the Kondo and the corresponding Anderson impurity models, in particular via scaling [6,7], large- $N$ expansions $[6,8,9]$ and the numerical renormalization group (NRG) [7,10-13]. All these techniques, whether for the soft-gap Anderson or Kondo models, show the existence of two distinct types of ground state, between which in general a nontrivial zero-temperature phase transition occurs at a finite value of the host-impurity coupling (or, equivalently in the Anderson model, at a finite value of the impurity onsite interaction, $U$ ): a weak coupling or local moment (LM) state in which the impurity spin remains unquenched; and a strong coupling (SC) state in which a Kondo effect is manifest, and whose properties - in particular for the so-called symmetric strong coupling state considered here - have been argued to represent a natural generalization of Fermi liquid physics (see especially [13]).

NRG studies in particular have devoted considerable attention to the spin- $\frac{1}{2}(N=2)$ particle-hole symmetric case, including thermodynamic [10-13] properties and, for the Anderson model, impurity spectral functions [12]. It is the symmetric spin- $\frac{1}{2}$ soft-gap Anderson model we consider here, with aims that are modest, and threefold. First, to consider briefly the non-interacting limit, $U=0$; second to establish rather general conditions upon the existence of a SC state at finite- $U$; and finally, to examine the problem explicitly at the simplest possible level-straight second-order perturbation theory in $U$.

There are two reasons for considering the non-interacting limit, simple though it is. First, and in contrast to the 'normal' Anderson model $(r=0)$, its behaviour is nontrivial and exemplifies much physics relevant to the interacting problem. An understanding of the impurity single-particle spectrum, $D_{0}(\omega)$, is sufficient to study the non-interacting limit, since both 'excess' thermodynamic properties induced by addition of the impurity, 
and local properties such as the impurity susceptibility, follow from a knowledge of it. As shown in section 3 , both LM and SC states arise in the $U=0$ limit, with characteristic and distinct signatures in both spectral and thermodynamic functions, as indeed known in part from the work of ref. [13]. LM states alone are found to occur for $r>1$ and SC states for $r<1$, whereas finite- $U$ NRG results $[12,13]$ show that LM states alone occur for $r>\frac{1}{2}$-an important contrast whose implications are considered in section 5 . The second reason for considering the non-interacting limit is prosaic: a knowledge of it underpins finite-order perturbation theory in $U$, as considered in section 5 .

In section 4 , focusing on the finite- $U$ impurity spectral function $D(\omega)$, we establish general conditions upon the interaction self-energy for the occurence of a SC state, from which follow in turn two results. First, that the low-frequency behaviour of the single-particle spectrum is $D(\omega) \sim|\omega|^{-r}$, which is precisely the spectral signature of the SC state found in NRG studies [12]. Second, and relatedly, that interactions have no influence in renormalizing the low- $\omega$ asymptotic behaviour of $D(\omega)$. In consequence, one obtains a conservation on $A(\omega)=|\omega|^{r} D(\omega)$ at the Fermi level, $\omega=0$ : for any $r$ where a SC state exists, $A(\omega=0)$ is pinned at its non-interacting value for all $U$, a result that generalizes to arbitrary $r$ the corresponding condition familiar for the $r=0$ Anderson model (see e.g.[1]).

In contrast to the $r=0$ Anderson model where the predictions of perturbation theory in $U$ about the non-interacting limit are well known (see e.g.[1]), the implications - and, indeed, general applicability - of a low-order perturbative treatment are not obvious for the soft-gap problem, and are considered in section 5 where we focus on the impurity spectral function. For $r>1$ second-order perturbation theory is found to recover the characteristic low- $\omega$ spectral signature of the LM state found in NRG studies [12], viz $D(\omega) \sim|\omega|^{r}$; and the resultant single-particle spectra are investigated in some detail. For $r<\frac{1}{2}$ we find the SC state is indeed perturbatively stable upon increasing $U$ from zero, and that the general conditions of section 4 for a SC state are satisfied at the second-order level; the resultant modified spectral functions $A(\omega)$ are also shown to bear a striking resemblance to that for the normal Anderson model, $r=0$, exhibiting in particular the emergence with increasing $U$ of a characteristic low-energy Kondo scale. For $\frac{1}{2}<r<1$ by contrast, we argue that finite-order perturbation theroy in $U$ about the non-interacting limit is simply inapplicable.

There is a second, 'hidden' reason why we consider low-order perturbation theory: to illustrate its limitations, despite its strengths, even for $r<\frac{1}{2}$ where the SC state is perturbatively continuable from the non-interacting limit. It is our belief that to describe analytically much of the underlying physics of the soft-gap Anderson modeland in particular to capture the transition between LM and SC (or generalized Fermi liquid) phases which render the problem of generic interest-requires, or at least invites, a new and inherently non-perturbative theoretical approach. We will turn to one such theory in a subsequent paper [14], for which the present work is in part a forerunner. 


\section{Background}

With the Fermi level taken as the origin of energy, the Hamiltonian for the spin- $\frac{1}{2}$ Anderson model is given in standard notation by

$$
\begin{aligned}
\hat{H} & =\hat{H}_{\text {host }}+\hat{H}_{\text {impurity }}+\hat{H}_{\text {hybridization }} \\
& =\sum_{\boldsymbol{k}, \sigma} \epsilon_{\boldsymbol{k}} \hat{n}_{\boldsymbol{k} \sigma}+\sum_{\sigma}\left(\epsilon_{i}+\frac{U}{2} \hat{n}_{i-\sigma}\right) \hat{n}_{i \sigma}+\sum_{\boldsymbol{k}, \sigma} V_{i \boldsymbol{k}}\left(c_{i \sigma}^{\dagger} c_{\boldsymbol{k} \sigma}+c_{\boldsymbol{k} \sigma}^{\dagger} c_{i \sigma}\right)
\end{aligned}
$$

with $\epsilon_{\boldsymbol{k}}$ the host dispersion, $V_{i \boldsymbol{k}}$ the hybridization and $\epsilon_{i}$ the impurity level; for the symmetric case considered here, $\epsilon_{i}=-\frac{U}{2}$ with $U$ the on-site interaction.

We consider the zero-temperature single-particle impurity Green function, $G(t)=$ $-\mathrm{i}\left\langle T\left\{c_{i \sigma}(t) c_{i \sigma}^{\dagger}\right\}\right\rangle$, with $G(\omega)$ expressible as

$$
G(\omega)=[\omega+\operatorname{i} \eta \operatorname{sgn}(\omega)-\Delta(\omega)-\Sigma(\omega)]^{-1}
$$

where the limit $\eta \rightarrow 0+$ is henceforth understood. Here $\Delta(\omega)$ is the hybridization function given by

$$
\begin{aligned}
\Delta(\omega) & =\sum_{\boldsymbol{k}} \frac{\left|V_{i \boldsymbol{k}}\right|^{2}}{\omega-\epsilon_{\boldsymbol{k}}+\mathrm{i} \eta \operatorname{sgn}(\omega)} \\
& =\Delta_{\mathrm{R}}(\omega)-\operatorname{isgn}(\omega) \Delta_{\mathrm{I}}(\omega)
\end{aligned}
$$

and we consider throughout a symmetric hybridization

$$
\Delta(\omega)=-\Delta(-\omega)
$$

(whose particular form is specified in §2.1). From particle-hole symmetry the Fermi level remains fixed at $\omega=0 \forall U \geq 0$, whence the impurity charge $n_{i}=\sum_{\sigma}\left\langle\hat{n}_{i \sigma}\right\rangle=1 \forall U$; and the interaction self-energy $\Sigma(\omega)$ is defined to exclude the trivial Hartree contribution of $(U / 2) n_{i}=U / 2$, which cancels $\epsilon_{i}=-U / 2 . \Sigma(\omega)$ may likewise be decomposed as

$$
\Sigma(\omega)=\Sigma_{\mathrm{R}}(\omega)-\operatorname{isgn}(\omega) \Sigma_{\mathrm{I}}(\omega)
$$

and the real parts of $\Sigma$ or $\Delta$ follow directly from the Hilbert transform

$$
F_{\mathrm{R}}(\omega)=\int_{-\infty}^{\infty} \frac{\mathrm{d} \omega_{1}}{\pi} F_{\mathrm{I}}\left(\omega_{1}\right) \mathrm{P}\left(\frac{1}{\omega-\omega_{1}}\right)
$$

with $\mathrm{F}=\Sigma$ or $\Delta$ as appropriate.

The single-particle impurity spectrum $D(\omega)=-\frac{1}{\pi} \operatorname{sgn}(\omega) \operatorname{Im} G(\omega)$ follows directly as

$$
D(\omega)=D^{\mathrm{p}}(\omega)+D^{\mathrm{b}}(\omega)
$$

where

$$
D^{\mathrm{b}}(\omega)=\frac{\left[\Delta_{\mathrm{I}}(\omega)+\Sigma_{\mathrm{I}}(\omega)\right] \pi^{-1}}{\left[\omega-\Delta_{\mathrm{R}}(\omega)-\Sigma_{\mathrm{R}}(\omega)\right]^{2}+\left[\eta+\Delta_{\mathrm{I}}(\omega)+\Sigma_{\mathrm{I}}(\omega)\right]^{2}}
$$

and

$$
D^{\mathrm{p}}(\omega)=\frac{\eta \pi^{-1}}{\left[\omega-\Delta_{\mathrm{R}}(\omega)-\Sigma_{\mathrm{R}}(\omega)\right]^{2}+\left[\eta+\Delta_{\mathrm{I}}(\omega)+\Sigma_{\mathrm{I}}(\omega)\right]^{2}} .
$$


Here $D^{\mathrm{b}}(\omega)$ refers to continuum (or 'band') excitations, while $D^{\mathrm{p}}(\omega)$ allows for the possibility of discrete states reflected in pole contributions.

The change in the density of states of the system due to addition of the impurity, $\Delta \rho(\omega)$, will also prove central to the subsequent analysis. It is given by

$$
\Delta \rho(\omega)=D(\omega)\left[1-\frac{\partial \Delta_{\mathrm{R}}(\omega)}{\partial \omega}\right]-\frac{1}{\pi} \operatorname{Re} G(\omega) \frac{\partial \Delta_{\mathrm{I}}(\omega)}{\partial \omega}
$$

and is calculable directly once the impurity $D(\omega)$ is known. Note that equation (2.9), while commonly derived explicitly for the non-interacting case (see eg [1]), is readily shown to hold generally for all $U$. From equation (2.7), $\Delta \rho(\omega)$ likewise separates into 'band' and 'pole' contributions,

$$
\Delta \rho(\omega)=\Delta \rho^{\mathrm{p}}(\omega)+\Delta \rho^{\mathrm{b}}(\omega)
$$

where in particular

$$
\Delta \rho^{\mathrm{p}}(\omega)=D^{\mathrm{p}}(\omega)\left[1-\frac{\partial \Delta_{\mathrm{R}}(\omega)}{\partial \omega}\right]
$$

\subsection{Hybridization function}

The hybridization function we consider, $\Delta_{\mathrm{I}}(\omega)\left(=\Delta_{\mathrm{I}}(-\omega)\right)$, is given by

$$
\Delta_{\mathrm{I}}(\omega)=\left\{\begin{array}{ccc}
\Delta_{0}\left(|\omega|-\frac{\delta}{2}\right)^{r} & : & \frac{\delta}{2}<|\omega|<D+\frac{\delta}{2} \\
0 & : & \text { otherwise }
\end{array}\right.
$$

with $r>0$. While a pure power-law hybridization is naturally not realistic on arbitrary energy scales, it captures in the simplest fashion the requisite low- $\omega$ behaviour; moreover, as for the $r=0$ Anderson model, one expects impurity properties to be controlled primarily by the low- $\omega$ behaviour and to be largely independent of the details of host band structure. In general the hybridization thus contains a gap of magnitude $\delta$, in which lies the Fermi level $\omega=0$; the 'normal' flat-band Anderson model is recovered as a special case of equation (2.11) with $r=0=\delta$. There are four energy scales in the

problem, namely $\delta, \Delta_{0}^{\frac{1}{1-r}}, D$ (the bandwidth) and $U$; we choose to rescale in terms of $\Delta_{0}^{\frac{1}{1-r}}$, defining for later purposes

$$
\tilde{\omega}=\frac{\omega}{\Delta_{0}^{\frac{1}{1-r}}}, \quad \tilde{D}=\frac{D}{\Delta_{0}^{\frac{1}{1-r}}}, \quad \tilde{U}=\frac{U}{\Delta_{0}^{\frac{1}{1-r}}} .
$$

From the Hilbert transform equation (2.6), $\Delta_{R}(\omega)=-\Delta_{R}(-\omega)$ is given by

$$
\Delta_{\mathrm{R}}(\omega)=\frac{2 \omega \Delta_{0}}{\pi} \int_{0}^{D} \mathrm{~d} \omega_{1} \frac{\omega_{1}^{r}}{\omega^{2}-\left(\omega_{1}+\frac{\delta}{2}\right)^{2}}
$$

where a principal value is henceforth understood. Notice from this that the wide-band limit $D \rightarrow \infty$, as commonly employed for the normal Anderson model $r=0=\delta$, can be taken only for $r<1$. 
For the gapped case, $\delta>0$, we shall need solely the behaviour of $\Delta_{\mathrm{R}}(\omega)$ for frequencies $|\omega| \ll \frac{\delta}{2}$ inside the gap. This is given from equation (2.13) by

$$
\Delta_{\mathrm{R}}(\omega)=-\omega \frac{2 \Delta_{0}}{\pi}\left[\frac{\delta}{2}\right]^{r-1} B(\delta ; r)+\mathrm{O}\left[\left(\frac{2 \omega}{\delta}\right)^{3}\right]
$$

where $B(\delta ; r) \geq 0$ is given by

$$
B(\delta ; r)=\int_{0}^{2 D / \delta} \mathrm{d} z \frac{z^{r}}{(1+z)^{2}} .
$$

Our primary focus in sections 4 and 5 will be the 'soft gap' case, $\delta=0$, where $\Delta_{\mathrm{I}}(\omega)=\Delta_{0}|\omega|^{r}$. For this case, rescaling of equation (2.13) yields

$$
\Delta_{\mathrm{R}}(\omega)=\operatorname{sgn}(\omega) \Delta_{0}|\omega|^{r} \frac{2}{\pi} \int_{0}^{D /|\omega|} \mathrm{d} y \frac{y^{r}}{\left(1-y^{2}\right)}
$$

which thus obeys the differential equation

$$
\frac{\partial \Delta_{\mathrm{R}}(\omega)}{\partial \omega}=\frac{r}{\omega} \Delta_{\mathrm{R}}(\omega)+\frac{2 \Delta_{0} D^{r-1}}{\pi}\left[1-\left(\frac{\omega}{D}\right)^{2}\right]^{-1} .
$$

Direct evaluation of equation (2.15) yields, for $|\omega|<D$,

$$
\begin{aligned}
\Delta_{\mathrm{R}}(\omega)= & -\operatorname{sgn}(\omega) \Delta_{0}\left\{\tan \left(\frac{\pi r}{2}\right)|\omega|^{r}+\frac{D^{r}}{\pi r}[F(1,-r ; 1-r ;|\omega| / D)\right. \\
& -F(1,-r ; 1-r ;-|\omega| / D)]\} \\
= & -\operatorname{sgn}(\omega) \Delta_{0}\left\{\tan \left(\frac{\pi r}{2}\right)|\omega|^{r}+\frac{2 D^{r}}{\pi(r-1)} \frac{|\omega|}{D}+\mathrm{O}\left[\left(\frac{|\omega|}{D}\right)^{3}\right]\right\}
\end{aligned}
$$

with $F(\alpha, \beta ; \gamma ; z)$ a Gauss hypergeometric function. Equation (2.17) encompasses the results given in [13] and applies for any $r \geq 0$, including $r=1$ for which the limit as $r \rightarrow 1$ of equation (2.17b) gives

$$
\Delta_{\mathrm{R}}(\omega)=\frac{2 \Delta_{0}}{\pi} \operatorname{sgn}(\omega)|\omega| \ln \left(\frac{|\omega|}{D}\right)+\mathrm{O}\left[\left(\frac{|\omega|}{D}\right)^{3}\right]: r=1
$$

showing the characteristic logarithmic behaviour that, as discussed in section 3, is indicative of the marginal nature of $r=1$ for $U=0$.

\section{Non-interacting limit}

As mentioned in section 1, the non-interacting problem is surprisingly rich: it contains already much underlying physics relevant to the interacting case and, for the soft-gap case in particular, gives rise to both SC and LM states as now considered.

The impurity spectrum $D_{0}(\omega)$ (with '0' referring to $U=0$ ) is the primary quantity, since the excess density of states $\Delta \rho_{0}(\omega)$ follows from it via equation (2.9). The low- $\omega$ behaviour of the latter determines in turn the low temperature behaviour of the change in thermodynamic properties due to addition of the impurity; the 'excess' total uniform 
spin susceptibility, specific heat and entropy being given trivially for non-interacting electrons by

$$
\begin{gathered}
\chi_{\mathrm{imp}}^{0}(T)=\frac{\left(g \mu_{B}\right)^{2}}{2 T} \int_{-\infty}^{\infty} \mathrm{d} \omega \Delta \rho_{0}(\omega) f(\omega)(1-f(\omega)) \\
C_{\mathrm{imp}}^{0}(T)=\frac{2}{T^{2}} \int_{-\infty}^{\infty} \mathrm{d} \omega \omega^{2} \Delta \rho_{0}(\omega) f(\omega)(1-f(\omega)) \\
S_{\mathrm{imp}}^{0}(T)=-2 \int_{-\infty}^{\infty} \mathrm{d} \omega \Delta \rho_{0}(\omega)[f(\omega) \ln f(\omega)+(1-f(\omega)) \ln (1-f(\omega))]
\end{gathered}
$$

where $f(\omega)$ is the Fermi function (with chemical potential $\mu=0$ for all $T$ due to particle-hole symmetry), and $k_{B} \equiv 1$ has been taken.

The band/continuum part of $\Delta \rho_{0}(\omega)$ is given from equations $(2.9,10)$ by

$$
\Delta \rho_{0}^{\mathrm{b}}(\omega)=D_{0}^{\mathrm{b}}(\omega)\left[1-\frac{\partial \Delta_{\mathrm{R}}(\omega)}{\partial \omega}\right]-\frac{1}{\pi} \operatorname{Re} G_{0}(\omega) \frac{\partial \Delta_{\mathrm{I}}(\omega)}{\partial \omega}
$$

with $D_{0}^{\mathrm{b}}(\omega)$ from $\mathrm{Eq}(2.8 \mathrm{a})$. For the gapless case $\delta=0$, a straightforward calculation using $\operatorname{Eq}(2.16)$ for $\partial \Delta_{\mathrm{R}} / \partial \omega$ gives a simple relation between $\Delta \rho_{0}^{\mathrm{b}}(\omega)$ and $D_{0}^{\mathrm{b}}(\omega)$ for $|\omega|<D:$

$$
\Delta \rho_{0}^{\mathrm{b}}(\omega)=D_{0}^{\mathrm{b}}(\omega) \frac{(1-r)}{q(\omega)} \quad: \quad \delta=0
$$

where

$$
q^{-1}(\omega)=\left[1+\frac{2 \Delta_{0} D^{r-1}}{\pi(r-1)} \frac{1}{1-\left(\frac{\omega}{D}\right)^{2}}\right] .
$$

The pole contributions, $D_{0}^{\mathrm{p}}(\omega)$ and $\Delta \rho_{0}^{\mathrm{p}}(\omega)$, are considered below. Here we simply note that for finite bandwidth, $D$, there are always such contributions outside the band, $|\omega|>D$. These however are of no importance to the problem, and are not considered explicitly in what follows where $|\omega|<D$ is implicit.

\subsection{Gapped case: LM state}

We consider first the case of an insulating host, with $\delta>0$. Since $\Delta_{\mathrm{I}}(\omega)=0$ for $|\omega|<\delta / 2$ inside the gap, it follows from equation (2.8b) (with $\Sigma=0$ ) that

$$
D_{0}^{\mathrm{p}}(\omega)=q \delta(\omega)
$$

with poleweight $q$ given by

$$
\begin{aligned}
& q^{-1}=\left[1-\left(\frac{\partial \Delta_{\mathrm{R}}(\omega)}{\partial \omega}\right)_{\omega=0}\right] \\
& =\quad 1+\frac{2 \Delta_{0}}{\pi}\left[\frac{\delta}{2}\right]^{r-1} B(\delta ; r)
\end{aligned}
$$

where equation (2.14) is used. From equation (2.8b) the band contribution $D_{0}^{\mathrm{b}}(\omega)$ naturally vanishes inside the gap, and behaves as $D_{0}^{\mathrm{b}}(\omega) \propto \Delta_{\mathrm{I}}(\omega) \sim(|\omega|-\delta / 2)^{r}$ close to 
the gap edges. $D_{0}(\omega)$ is thus dominated by the discrete state at the Fermi level, $\omega=0$, as too is $\Delta \rho_{0}(\omega)$ : from equations $(2.10 \mathrm{~b})$ and $(3.4,5)$,

$$
\Delta \rho_{0}^{\mathrm{p}}=\delta(\omega)
$$

whose poleweight of unity reflects a 'whole' single extra state at the Fermi level induced by addition of the impurity.

These features - a whole excess state at the Fermi level, with non-vanishing weight on the impurity - are the hallmark of the LM state for $U=0$. They naturally control the low- $T$ excess thermodynamic functions, given from equations (3.1) by

$$
\begin{aligned}
& \chi_{\text {imp }}^{0}(T)=\frac{\left(g \mu_{B}\right)^{2}}{8 k T}=\frac{1}{2} \chi_{\text {Curie }}(T) \\
& S_{\text {imp }}^{0}(0)=\ln 4
\end{aligned}
$$

with corrections that are thermally activated (as too is $C_{\mathrm{imp}}^{0}(T)$ ). The residual entropy of $\ln 4$ is physically obvious: the excess Fermi level state has four occupancies - empty, $\uparrow$-spin or $\downarrow$-spin occupied and doubly occupied - with equal a priori probabilities for $U=0$. This is also why $\chi_{\mathrm{imp}}^{0}(T)$ is half a Curie law: only half of the four occupancies, the singly occupied states, are paramagnetically active. This situation will of course change immediately for any $U>0$ where doubly occupied (and hence empty) spin configurations are suppressed, producing instead $\chi_{\text {imp }}(T)=\chi_{\text {curie }}(T)$ as $T \rightarrow 0$, and $S_{\text {imp }}(0)=\ln 2$.

The gapless case, $\delta=0$, is considered in sections $3.2,3$, but first we ask what happens as $\delta \rightarrow 0$. From equation (2.14),

$$
B(\delta ; r)=\frac{\pi r}{\sin (\pi r)}+\frac{1}{(r-1)} \frac{x^{1-r}}{(1+x)} F(1,-r ; 2-r ;-x)
$$

with

$$
x=\frac{\delta}{2 D} .
$$

From equation (3.5b), the behaviour of the poleweight $q$ for $\delta / 2 D \ll 1$ can thus be obtained. For $r>1$ one finds

$$
q^{-1}=\left[1+\frac{2 \Delta_{0} D^{r-1}}{\pi(r-1)}\right]+\mathrm{O}\left(\delta^{r-1} ; \delta\right) \quad: r>1
$$

which remains finite as $\delta \rightarrow 0$ (where the resultant $q=q(\omega=0)$, from equation (3.3b)), showing the persistence of the LM state for $r>1$ when $\delta=0$. For $r<1$ by contrast,

$$
q^{-1}=1+\frac{2 r}{\sin (\pi r)} \Delta_{0} D^{r-1}\left[\frac{\delta}{2 D}\right]^{r-1}+\mathrm{O}(1) \quad: \quad r<1
$$

which diverges as $\delta \rightarrow 0$ (as too does the marginal case of $r=1$, where $\left.q^{-1} \sim 1 / \ln (2 D / \delta)\right)$. For $r<1$, the LM state does not therefore survive closure of the gap. 


\section{$\mathbf{D}_{0}(\omega)$}
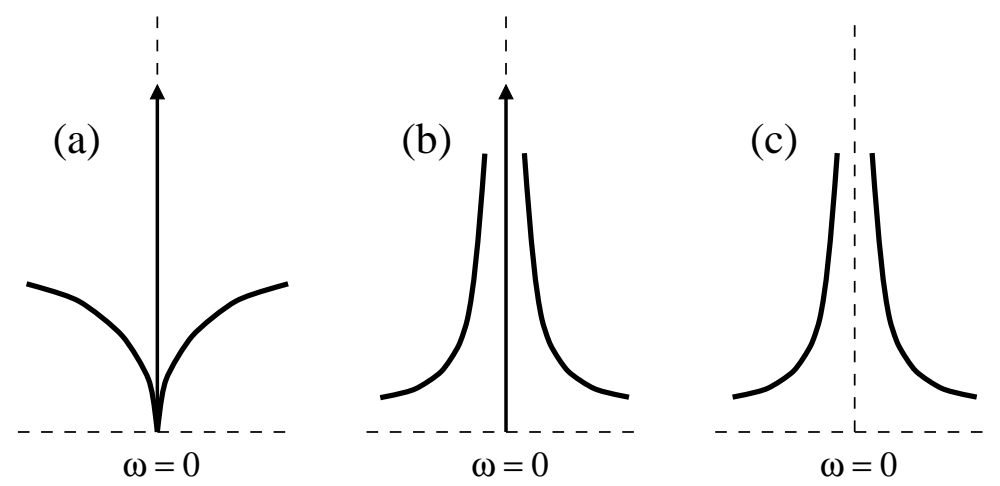

Figure 1. Schematic of non-interacting impurity spectra $D_{0}(\omega)$ for (a) LM state, $r>2$; (b) LM state, $1<r<2$; (c) SC state, $r<1$.

\subsection{Gapless case: $r>1$, LM state}

For $r>1$, the LM state indeed persists with the gap shut, $\delta=0$ : since $\Delta_{\mathrm{I}}(\omega) \sim|\omega|^{r}$ decays to zero as $\omega \rightarrow 0$ more rapidly than $\Delta_{\mathrm{R}}(\omega) \sim \mathrm{O}(|\omega|)$ (see equation (2.17b)), equation $(2.8 \mathrm{~b})$ yields directly $D_{0}^{\mathrm{p}}=q \delta(\omega)$ with $q$ given by equation (3.5a) and hence equation (3.9) with $\delta=0$. Likewise, from equation (2.10b), $\Delta \rho_{0}^{\mathrm{p}}(\omega)=\delta(\omega)$ contains a 'whole' extra state.

In contrast to the gapped case however, $D_{0}^{\mathrm{b}}(\omega)$ now extends down to $\omega=0$. From equations (2.8a) and (2.17b) one obtains

$$
D_{0}^{\mathrm{b}}(\omega)=\frac{\Delta_{0} q^{2}}{\pi}|\omega|^{r-2}+\mathrm{O}\left(|\omega|^{2 r-3}\right)
$$

diverging as $\omega \rightarrow 0$ for $1<r<2$ and vanishing for $r>2$, as found in [13]. The full $D_{0}(\omega)$ consists of course of both pole and band contributions; it is illustrated schematically in figure 1. From equations (3.3) and (3.11) the low- $\omega$ behaviour of $\Delta \rho_{0}^{\mathrm{b}}(\omega)$ is

$$
\Delta \rho_{0}^{\mathrm{b}}(\omega)=\frac{\Delta_{0} q}{\pi}(1-r)|\omega|^{r-2}+\mathrm{O}\left(|\omega|^{2 r-3} ;|\omega|^{r}\right) .
$$

The excess thermodynamic properties follow directly via equation (3.1): $S_{\mathrm{imp}}^{0}(0)=\ln 4$ again, while

$$
\begin{aligned}
& \chi_{\text {imp }}^{0}(T)=\frac{1}{2} \chi_{\text {curie }}(T)+c^{\prime} T^{r-2}+\mathrm{O}\left(T^{2 r-3} ; T^{r}\right) \\
& C_{\text {imp }}^{0}(T)=d^{\prime} T^{r-1}+\mathrm{O}\left(T^{2(r-1)} ; T^{1+r}\right)
\end{aligned}
$$

where $c^{\prime}$ and $d^{\prime}$ are negative constants (whose sign reflects the low- $\omega$ 'depletion' of $\Delta \rho_{0}^{\mathrm{b}}(\omega)$ - the coefficient of the leading $|\omega|^{r-2}$ term in equation (3.12) being negative for $r>1$ ). Not surprisingly, and in part for the reasons discussed in section 3.1, the behaviour equation (3.13) characteristic of the $U=0$ limit differs from that found by NRG for the $U>0$ LM regime [12]: $\chi_{\text {imp }}(T)=\chi_{\text {Curie }}(T)+c_{3}^{\prime} T^{r-1}$ and $C_{\text {imp }}(T) \propto T^{r}$. 


\subsection{Gapless case: $r<1$, SC state}

From equations (2.8a) and (2.17b), a simple calculation gives the low- $\omega$ behaviour of the continuum contribution to $D_{0}(\omega)$, viz

$$
D_{0}^{\mathrm{b}}(\omega)=\frac{|\omega|^{-r}}{\pi \Delta_{0}\left(1+\beta^{2}\right)}\left[1-\frac{\beta}{1+\beta^{2}} \frac{2}{\Delta_{0} q(0)}|\omega|^{1-r}\right]+\mathrm{O}\left(|\omega|^{2-3 r}\right)
$$

where

$$
\beta=\tan \left(\frac{\pi}{2} r\right)
$$

$\Delta \rho_{0}^{\mathrm{b}}(\omega)$ follows directly from equation (3.3a) and has the same leading asymptotics as $D_{0}^{\mathrm{b}}(\omega)$.

Now consider $D_{0}^{\mathrm{p}}(\omega)$, given from equation $(2.8 \mathrm{~b})$ with $\Sigma=0$. From equation $(2.17 \mathrm{~b})$ the leading low- $\omega$ behaviour of $\Delta_{\mathrm{R}}(\omega)$ for $0<r<1$ may be cast as

$$
\Delta_{\mathrm{R}}(\omega)=-\operatorname{sgn}(\omega) \tan \left(\frac{\pi}{2} r\right) \Delta_{\mathrm{I}}(\omega) \quad: \quad \omega \rightarrow 0
$$

whence

$$
D_{0}^{\mathrm{p}}(\omega) \equiv L\left(\Delta_{\mathrm{I}} ; r\right)
$$

with $L(x ; \lambda)$ (used again in section 4 ) defined generally by

$$
\begin{aligned}
L(x ; \lambda) & =\frac{\eta \pi^{-1}}{\left[\tan \left(\frac{\pi}{2} \lambda\right) x\right]^{2}+[\eta+x]^{2}} \quad: \quad \eta \rightarrow 0+ \\
& =\frac{\lambda}{\tan \left(\frac{\pi}{2} \lambda\right)} \delta(x) .
\end{aligned}
$$

Since $\Delta_{\mathrm{I}}(\omega)=\Delta_{0}|\omega|^{r}$, it follows that

$$
D_{0}^{\mathrm{p}}(\omega)=\frac{1}{\Delta_{0} \tan \left(\frac{\pi}{2} r\right)}|\omega|^{1-r} \delta(\omega) \quad \equiv 0
$$

i.e. there is no pole contribution to $D_{0}(\omega)$ itself, consistent with section (3.1) as $\delta \rightarrow 0$. Hence $D_{0}(\omega) \equiv D_{0}^{\mathrm{b}}(\omega)$ is given by equation (3.14), with $D_{0}(\omega) \sim|\omega|^{-r}$ as found in [13] (and illustrated schematically in figure 1c).

But from equation $(2.10 \mathrm{~b}), \Delta \rho_{0}^{\mathrm{p}}(\omega)$ is given in contrast by

$$
\begin{aligned}
\Delta \rho_{0}^{\mathrm{p}}(\omega) & =D_{0}^{\mathrm{p}}(\omega)\left[1+\Delta_{0} r \tan \left(\frac{\pi}{2} r\right)|\omega|^{r-1}\right] \\
& =r \delta(\omega)
\end{aligned}
$$

(via equation (3.18)), and thus contains a $\delta$-function contribution of weight $r$, as well known from the work of $[10,13]$ where the result was obtained from analysis of the phase shift. This behaviour is the characteristic of the SC state, and suggests the interpretation [13] that a fraction $r$ of a conduction electron occupies the decoupled excess state at the Fermi level, the remaining fraction $1-r$ being absorbed into the resonant continuum centred on $\omega=0$. And note again that, in contrast to the LM state, the excess level has no weight on the impurity itself: $D_{0}^{\mathrm{p}}(\omega) \equiv 0$. 
Magnetic impurities in gapless Fermi systems: perturbation theory

Excess thermodynamic properties follow directly via equation (3.1), namely

$$
\begin{aligned}
& \chi_{\text {imp }}^{0}(T)=\frac{r}{2} \chi_{\text {curie }}(T)+c T^{-r}+\mathrm{O}\left(T^{1-2 r}\right) \\
& C_{\text {imp }}^{0}(T)=d T^{1-r}+\mathrm{O}\left(T^{2(1-r)}\right) \\
& S_{\text {imp }}^{0}(T)=2 r \ln 2+e T^{1-r}+\mathrm{O}\left(T^{2(1-r)}\right)
\end{aligned}
$$

where $c, d$ and $e$ are positive constants. These are worth noting for comparison to NRG results obtained for the $U>0$ SC phase, namely [10-13]

$$
\begin{aligned}
& \chi_{\text {imp }}(T)=\frac{r}{2} \chi_{\text {Curie }}(T)+c_{1}^{\prime} T^{-r}+c_{2}^{\prime} T^{-2 r} \\
& C_{\text {imp }}(T) \propto T^{1-r} \\
& S_{\text {imp }}(T)=2 r \ln 2+e^{\prime} T^{1-r} .
\end{aligned}
$$

It is well known $[10,13]$ that the leading low- $T$ behaviour of $\chi_{\mathrm{imp}}$ and $S_{\mathrm{imp}}$ for the SC phase - viz $\frac{r}{2} \chi_{\text {Curie }}$ and $2 r \ln 2$ respectively — are given precisely by the $U=0$ limit result. But it is striking to note that the leading $T$-dependences of $C_{\mathrm{imp}}(T)$ and $\Delta S_{\mathrm{imp}}(T)$, namely $T^{1-r}$, are also inherent to the non-interacting limit, as too is the $T^{-r}$ correction to $\chi_{\mathrm{imp}}$. Only the $T^{-2 r}$ contribution to the NRG $\chi_{\mathrm{imp}}$ - which applies only for $r<\frac{1}{2}$ where the SC phase arises for $U>0$ - is absent in the non-interacting limit.

\subsection{Local impurity susceptibility}

The 'excess' $\chi_{\mathrm{imp}}^{0}(T)$ discussed above refers to the change in the total uniform spin susceptibility of the system induced by addition of the impurity. Here we comment briefly on the local impurity susceptibility $\chi_{i i}(T)$, which has also been studied via NRG $[10,13]$. It is defined by

$$
\chi_{i i}(T)=-\left.g \mu_{\mathrm{B}} \frac{\partial\left\langle\hat{S}_{i z}\right\rangle}{\partial h}\right|_{h=0}
$$

with $\hat{S}_{i z}$ referring to the impurity spin and $h$ a magnetic field acting solely on the impurity; and is given in standard notation by

$$
\chi_{i i}(T)=\left(g \mu_{\mathrm{B}}\right)^{2} \int_{0}^{\beta} \mathrm{d} \tau\left\langle\hat{S}_{i z}(\tau) \hat{S}_{i z}\right\rangle
$$

with $\beta=1 / T$. In the non-interacting limit, $\chi_{i i}^{0}(T)$ is trivially evaluated and given by

$$
\chi_{i i}^{0}(T)=\frac{\left(g \mu_{\mathrm{B}}\right)^{2}}{2} \int_{-\infty}^{\infty} \mathrm{d} \omega_{1} \int_{-\infty}^{\infty} \mathrm{d} \omega_{2} \frac{D_{0}\left(\omega_{1}\right) D_{0}\left(\omega_{2}\right)}{\omega_{1}-\omega_{2}} f\left(\omega_{1}\right)\left[1-f\left(\omega_{2}\right)\right]\left[e^{\beta\left(\omega_{1}-\omega_{2}\right)}-1\right] .
$$

Separating $D_{0}(\omega)=q \delta(\omega)+D_{0}^{\mathrm{b}}(\omega)$, and using particle-hole symmetry, a simple calculation gives

$$
\begin{aligned}
\frac{\chi_{i i}^{0}(T)}{\left(g \mu_{\mathrm{B}}\right)^{2}}=\frac{q^{2}}{8 T} & +\frac{q}{2} \int_{-\infty}^{\infty} \mathrm{d} \omega \frac{D_{0}^{\mathrm{b}}(\omega)}{\omega} \tanh \left(\frac{\omega}{2 T}\right) \\
& +\frac{1}{2} \int_{-\infty}^{\infty} \mathrm{d} \omega_{1} \int_{-\infty}^{\infty} \mathrm{d} \omega_{2} D_{0}^{\mathrm{b}}\left(\omega_{1}\right) D_{0}^{\mathrm{b}}\left(\omega_{2}\right) \frac{\left[f\left(\omega_{2}\right)-f\left(\omega_{1}\right)\right]}{\omega_{1}-\omega_{2}} .
\end{aligned}
$$


For the gapless LM regime $r>1$, where the poleweight $q(r) \neq 0$ is given by equation (3.9) (with $\delta=0$ ), the low- $T$ behaviour of $\chi_{i i}^{0}(T)$ is thus

$$
\chi_{i i}^{0}(T)=\frac{q^{2}}{2} \chi_{\text {Curie }}(T) \quad: r>1
$$

with leading corrections $\mathrm{O}\left[\min \left(T^{r-2}, 1\right)\right]$ arising from the second term of equation $(3.24)$. Hence, as expected for a LM state and mirrored also in $\chi_{\text {imp }}^{0}(T)$ equation (3.13a), the impurity spin remains unquenched; although $\chi_{i i}^{0}(T) / \chi_{\text {imp }}^{0}(T)=q^{2}<1$ as $T \rightarrow 0$, reflecting the fact that the 'whole' excess level induced by the impurity has only partial weight on the impurity itself.

For the SC phase $r<1$, by contrast, the excess level has no overlap on the impurity, $q=0$. Only the final term in equation (3.24) survives, and using equation (3.14a) is $\mathrm{O}(1)$ for $r<\frac{1}{2}$ and $\mathrm{O}\left(T^{1-2 r}\right)$ for $\frac{1}{2}<r<1$; hence, in particular,

$$
\lim _{T \rightarrow 0} T \chi_{i i}^{0}(T)=0 \quad: 0 \leq r<1 .
$$

As for the normal $(r=0)$ Anderson model, the impurity spin is thus locally quenched in the entire SC regime $r<1$; in contrast, for obvious reasons, to the behaviour of $\chi_{\text {imp }}^{0}$, equation (3.20a).

The above behaviour - complete (SC) versus incomplete (LM) quenching of the impurity spin - is also found in NRG studies of the soft-gap Kondo model [11,13]; and the total spin quenching symptomatic of the SC phase is one reason why it may be regarded [13] as a natural generalization of conventional Fermi liquid physics.

We have seen that even the non-interacting limit contains both LM and SC states, whose characteristics are reflected in the behaviour of the impurity spectrum $D_{0}(\omega)$ by whether (LM) or not (SC) there is a $\delta$-function contribution at the Fermi level-and hence in turn in $\Delta \rho_{0}(\omega)$ and resultant thermodynamic functions. One final point should however be noted: the $U=0$ 'phase diagram' consists of LM states for $r>1$ and SC states for $r<1$. This is in contrast to what is found by NRG for $U>0$ [12,13], where $\mathrm{SC}$ states arise only for $r<\frac{1}{2}$ and where only LM states occur for $r>\frac{1}{2}$. We consider the implications of this further in section 5 .

\section{4. $U>0$ : conditions for $\mathrm{SC}$ phase}

For non-vanishing interaction strengths, $U$, we now seek conditions under which, upon increasing $U$ from zero, a SC state will persist; (only the gapless problem, and for $r<1$, need be considered, since for $r>1$ the $U=0$ ground state is a local moment one). Employing a phase shift analysis that parallels Nozières' Fermi liquid description of the 'normal' Kondo effect [15], Chen and Jayaprakash [10] have argued that the SC state is characterized generally by

$$
\Delta \rho^{\mathrm{p}}(\omega)=r \delta(\omega) \quad: \mathrm{SC}
$$

- precisely as for the non-interacting limit. 
The question now is: under what conditions upon the self-energy $\Sigma(\omega)$ will this arise? $\Delta \rho^{\mathrm{p}}(\omega)$ is given by equation $(2.10 \mathrm{~b})$ with $D^{\mathrm{p}}(\omega)$ from equation $(2.8 \mathrm{~b})$. Since $\Delta_{\mathrm{I}}$ and $\Sigma_{\mathrm{I}}$ are non-negative and, for $|\omega|<D, \Delta_{\mathrm{I}}(\omega)=\Delta_{0}|\omega|^{r}$ vanishes only for $\omega=0$, the only $\delta$-funtion contribution that could arise in $\Delta \rho^{\mathrm{p}}(\omega)$ for $|\omega|<D$ is of course for $\omega=0$. Hence only the low- $\omega$ behaviour of $\Delta$ and $\Sigma$ is relevant, and $\Delta \rho^{\mathrm{p}}(\omega)$ is given by (cf equation(3.19a))

$$
\Delta \rho^{\mathrm{P}}(\omega)=D^{\mathrm{P}}(\omega)\left[1+\Delta_{0} r \tan \left(\frac{\pi}{2} r\right)|\omega|^{r-1}\right]
$$

with

$$
D^{\mathrm{p}}(\omega) \equiv \frac{\eta \pi^{-1}}{\left[\Delta_{\mathrm{R}}(\omega)+\Sigma_{\mathrm{R}}(\omega)\right]^{2}+\left[\eta+\Delta_{\mathrm{I}}(\omega)+\Sigma_{\mathrm{I}}(\omega)\right]^{2}}
$$

(where the 'bare' $\omega$ contribution to equation (2.8b) can again be neglected since, for $r<1$, it is subdominant to $\left.\Delta_{\mathrm{R}}(\omega) \sim|\omega|^{r}\right)$. Notice also that if the low- $\omega$ behaviour of $\Sigma_{\mathrm{I}}(\omega)$ is of form

$$
\Sigma_{\mathrm{I}}(\omega)=\alpha|\omega|^{\lambda} \quad: \quad \omega \rightarrow 0
$$

with $-1<\lambda<1$, where $\alpha \equiv \alpha(U)$ is a (necessarily positive) constant, then the corresponding low- $\omega$ behaviour of $\Sigma_{\mathrm{R}}(\omega)$ follows directly from the Hilbert transform equation (2.6) as

$$
\Sigma_{\mathrm{R}}(\omega)=-\operatorname{sgn}(\omega) \tan \left(\frac{\pi}{2} \lambda\right) \Sigma_{\mathrm{I}}(\omega) \quad: \quad \omega \rightarrow 0 .
$$

If $\Sigma_{\mathrm{I}}(\omega)$ and hence $\Sigma_{\mathrm{R}}(\omega)$ decay to zero as $\omega \rightarrow 0$ more rapidly than $\Delta_{\mathrm{I}}(\omega)$-i.e. if $\Sigma_{\mathrm{I}}(\omega)$ is of form equation (4.3a) with $r<\lambda$-then, trivially, the low- $\omega$ behaviour of equation $(4.2 \mathrm{~b})$ for $D^{\mathrm{p}}(\omega)$ is precisely that of the $U=0$ limit, i.e. $D^{\mathrm{p}}(\omega)=L\left(\Delta_{\mathrm{I}} ; r\right)$ (see equation (3.16)). Hence, as in equations (3.17) ff, $\Delta \rho^{\mathrm{p}}(\omega)=r \delta(\omega)$ arises. If by contrast $\Sigma_{\mathrm{I} / \mathrm{R}}$ dominate the low- $\omega$ behaviour of equation $(4.2 \mathrm{~b})$-i.e. if $\Sigma_{\mathrm{I}}(\omega)$ is of form equation (4.3a) with $\lambda<r$-then from equations (4.3) and (3.17), $D^{\mathrm{p}}(\omega)=L\left(\Sigma_{\mathrm{I}} ; \lambda\right)$; from equation (4.2a) it then follows that $\Delta \rho^{\mathrm{p}}(\omega) \sim|\omega|^{r-\lambda} \delta(\omega) \equiv 0$ since $\lambda<r$, i.e. there is no pole contribution to $\Delta \rho(\omega)$. Finally, if the low- $\omega$ behaviour of $\Sigma_{\mathrm{I} / \mathrm{R}}$ is the same as that of $\Delta_{\mathrm{I} / \mathrm{R}}$, viz $\lambda=r$ in equations (4.3), then a directly analogous calculation gives $\Delta \rho^{\mathrm{p}}(\omega)=r /\left[1+\alpha(U) / \Delta_{0}\right] \delta(\omega)$; i.e. a $\delta$-function contribution but with a $U$-dependent weight that is less than $r$.

The question posed above is thus answered: for $\Delta \rho^{\mathrm{p}}(\omega)=r \delta(\omega)$ to arise, and thus a SC state to be realized for $U>0, \Sigma_{\mathrm{I}}(\omega)$ and hence $\Sigma_{\mathrm{R}}(\omega)$ must decay to zero as $\omega \rightarrow 0$ more rapidly that $|\omega|^{r}$, i.e.

$$
\Sigma_{\mathrm{I}}(\omega) \stackrel{\omega \rightarrow 0}{\sim} \alpha|\omega|^{\lambda} \quad: \quad r<\lambda
$$

This has important implications for the low- $\omega$ behaviour of the impurity singleparticle spectrum $D(\omega)\left(\equiv D^{\mathrm{b}}(\omega)\right)$, since from equation $(2.8 \mathrm{a})$ it follows that the low- $\omega$ asymptotic behaviour of $D(\omega)$ is precisely that of $D_{0}(\omega)$, whence in particular

$$
D(\omega) \stackrel{\omega \rightarrow 0}{\sim}|\omega|^{-r}
$$


as indeed found for the SC phase in NRG studies of the impurity spectrum [12]. More importantly, using

$$
\lim _{\omega \rightarrow 0}|\omega|^{r} D(\omega)=\lim _{\omega \rightarrow 0}|\omega|^{r} D_{0}(\omega)
$$

together with equation (3.14), and defining $A(\omega)=|\omega|^{r} D(\omega)$, it follows that

$$
\pi \Delta_{0}\left[1+\tan ^{2}\left(\frac{\pi}{2} r\right)\right] A(\omega=0)=1 .
$$

We believe this to be significant. For the 'normal' Anderson model, $r=0$, it recovers the well known result (see e.g.[1]), normally viewed as a consequence of the Friedel sum rule, that the impurity single particle spectrum is pinned at the Fermi level $\omega=0$, i.e. that $D(\omega=0)=1 / \pi \Delta_{0}$ (in this case for all $U$ ). Equation (4.7) represents a generalization of this pinning condition to arbitrary $r$ where a SC state obtains, whose continuity in $r$ reflects the fact that interactions have no influence in renormalizing the asymptotic behaviour of $D(\omega)$ as $\omega \rightarrow 0$; and which is entirely consistent with the conlusions of Gonzalez-Buxton and Ingersent [13] from NRG studies that the SC state embodies a natural generalization of standard Fermi liquid physics. The extent to which equation (4.7) is captured in practice should also provide a good test of the accuracy of NRG calculations at low frequencies, and will be discussed elsewhere; moreover the generalized pinning condition will prove central to our local moment approach to the problem, as will be discussed in a subsequent paper [14].

One important question is not of course answered by the above considerations: for what range of $r$ will the condition equation (4.4) for a SC state actually arise? NRG calculations give $r<\frac{1}{2}$ for the SC state [10-13]. We examine this question in the following section within the framework of second order PT in $U$, together with a corresponding analysis of the evolution with $U$ of the single-particle spectra appropriate to the LM state.

\section{Perturbation theory in $U$}

Low order perturbation theory in $U$ about the non-interacting limit is probably the simplest and certainly the most conventional approach to the problem. For the 'normal' Anderson model, $r=0$, its predictions are of course well known (see e.g.[1]): while restricted by contruction to weak coupling interactions $U$, and thus incapable of capturing strong coupling 'Kondo' asymptotics, it generates order by order characteristic Fermi liquid behaviour, in particular that $\Sigma_{\mathrm{I}} \sim \mathrm{O}\left(\omega^{2}\right)$ and that the impurity spectrum is pinned at the Fermi level, $D(0)=1 / \pi \Delta_{0}$; and the single-particle spectrum evolves continuously upon increasing $U$ from the non-interacting limit, in accordance with the fact that the normal Anderson model is a Fermi liquid for all $U / \Delta_{0} \geq 0$. For $r>0$ by contrast the implications of a low order perturbative treatment - and even whether such is in general applicable - are far from obvious, and are considered here at the simplest second order level. 


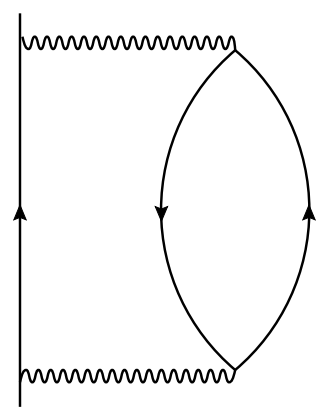

Figure 2. Second order self-energy diagram, with bare $(U=0)$ impurity propagators denoted by solid lines and the on-site impurity $U$ by wavy lines.

The familiar second order self-energy diagram is shown in figure 2 and may be written as

$$
\Sigma(\omega)=U^{2} \int_{-\infty}^{\infty} \frac{\mathrm{d} \omega_{1}}{2 \pi i} 0 \Pi\left(\omega_{1}\right) G_{0}\left(\omega_{1}+\omega\right)
$$

where $G_{0}(\omega)$ is the non-interacting impurity Green function with spectral representation

$$
G_{0}(\omega)=\int_{-\infty}^{\infty} \mathrm{d} \omega_{1} \frac{D_{0}\left(\omega_{1}\right)}{\omega-\omega_{1}+i \eta \operatorname{sgn}(\omega)} .
$$

The 'polarization bubble' ${ }^{0}(\omega)$ is given by

$$
\begin{aligned}
0 \Pi(\omega) & =i \int_{-\infty}^{\infty} \frac{\mathrm{d} \omega_{1}}{2 \pi} G_{0}\left(\omega_{1}\right) G_{0}\left(\omega_{1}-\omega\right) \\
& =\Pi \Pi(-\omega)
\end{aligned}
$$

(where the latter follows from a trivial change of variables in equation (5.3a)); its spectral representation is

$$
0 \Pi(\omega)=\int_{-\infty}^{\infty} \frac{\mathrm{d} \omega_{1}}{\pi} \frac{\operatorname{Im} \Pi \Pi\left(\omega_{1}\right) \operatorname{sgn}\left(\omega_{1}\right)}{\omega_{1}-\omega-i \eta \operatorname{sgn}(\omega)} .
$$

From equation (5.1), using (5.2) and (5.4) together with $D_{0}(\omega)=D_{0}(-\omega)$ (particlehole symmetry), a straightforward calculation gives

$$
\Sigma_{\mathrm{I}}(\omega)=U^{2} \int_{0}^{|\omega|} d \omega_{1} \operatorname{Im} \Pi\left(\omega_{1}\right) D_{0}\left(\omega_{1}-|\omega|\right)
$$

which is thus readily calculable from a knowledge of $D_{0}(\omega)$ (section 3) and $9 \Pi(\omega)$. Similarly, using equation (5.2) again, a directly analogous calculation yields

$$
\frac{1}{\pi} \operatorname{Im} \Pi \Pi(\omega)=\int_{0}^{|\omega|} \mathrm{d} \omega_{1} D_{0}\left(\omega_{1}\right) D_{0}\left(\omega_{1}-|\omega|\right) \quad \geq 0 .
$$

Note that, using equation (5.6), equation (5.5) implies $\Sigma_{\mathrm{I}}(\omega) \geq 0$ as required by analyticity. Equations (5.5) and (5.6) are the basic equations to be analyzed, as now considered for the gapless problem $(\delta=0)$.

Separating $D_{0}(\omega)$ into pole and band contributions, and using $D_{0}^{\mathrm{p}}(\omega)=q \delta(\omega)$, equation (5.6) reduces for $|\omega|<D$ to

$$
\frac{1}{\pi} \operatorname{Im} 9 \Pi(\omega)=\frac{1}{2} q^{2} \delta(\omega)+q D_{0}^{\mathrm{b}}(\omega)+\int_{0}^{|\omega|} \mathrm{d} \omega_{1} D_{0}^{\mathrm{b}}\left(\omega_{1}\right) D_{0}^{\mathrm{b}}\left(\omega_{1}-|\omega|\right) .
$$


This encompasses both $r>1$ where $q \neq 0$ is given from equation (3.9) with $\delta=0$; and $r<1$ where $q=0$ (section 3.3) and only the 'band-band' contribution to $\operatorname{Im}{ }^{9} \Pi(\omega)$ survives. [We add in passing that pole-contributions to $D_{0}^{\mathrm{p}}(\omega)$ from outside the band, $|\omega|>D$, generate spectral contributions to $\operatorname{Im}{ }^{0} \Pi(\omega)$ for $D<|\omega|<2 D$; as we shall be concerned only with the low- $\omega$ behaviour of $\operatorname{Im} \Pi \Pi(\omega)$ and hence $\Sigma_{\mathrm{I}}(\omega)$, we refrain from showing these, although they are fully included in numerical calculations where they are necessary to ensure the correct normalization of $D(\omega)$.]

The low- $\omega$ asymptotics of $\operatorname{Im}{ }^{9} \Pi(\omega)$ follow from equation (5.7) using the results of section 3 for $D_{0}(\omega)$. For $r>1$ we obtain

$$
\frac{1}{\pi} \operatorname{Im} 9 \Pi(\omega) \stackrel{\omega \rightarrow 0}{\sim} \frac{1}{2} q^{2} \delta(\omega)+q D_{0}^{\mathrm{b}}(\omega)+\mathrm{O}\left(|\omega|^{2 r-3}\right) \quad: \quad r>1
$$

where the $\mathrm{O}\left(|\omega|^{2 r-3}\right)$ corrections arise from the band-band piece of equation (5.7) and diverge less rapidly as $\omega \rightarrow 0$ than the leading $|\omega|^{r-2}$ behaviour of $D_{0}^{\mathrm{b}}(\omega)$ (equation (3.11)). For $r<1$ by contrast, only the band-band contribution to equation (5.7) survives, and a simple calculation using equation (3.14) gives the leading low- $\omega$ behaviour

$$
\frac{1}{\pi} \operatorname{Im} 9 \Pi(\omega) \stackrel{\omega \rightarrow 0}{\sim} C(r)|\omega|^{1-2 r} \quad: \quad r<1
$$

with $C>0$ given by

$$
\begin{aligned}
& C(r)=\frac{\cos ^{4}\left(\frac{\pi}{2} r\right)}{\pi \Delta_{0}} \int_{0}^{1} \mathrm{~d} y y^{-r}(1-y)^{-r} \\
& =\quad \frac{\cos ^{4}\left(\frac{\pi}{2} r\right)}{\Delta_{0}} \frac{2^{2 r-1}}{\sqrt{\pi}} \frac{\Gamma(1-r)}{\Gamma\left(\frac{3}{2}-r\right)} .
\end{aligned}
$$

These results may now be used in equation (5.5) to determine the crucial low- $\omega$ asymptotics of $\Sigma_{\mathrm{I}}(\omega)$, as now considered separately for $r>1$ and $r<1$.

\section{1. $r>1:$ LM state}

From equations (5.5) and (5.8) the low- $\omega$ behaviour of $\Sigma_{\mathrm{I}}(\omega)$ is given by

$$
\begin{aligned}
\Sigma_{\mathrm{I}}(\omega) & =\frac{\pi U^{2} q^{2}}{4}\left[q \delta(\omega)+3 D_{0}^{\mathrm{b}}(\omega)+\mathrm{O}\left(|\omega|^{2 r-3}\right)\right] \\
& =\frac{\pi U^{2} q^{3}}{4} \delta(\omega)+\Sigma_{\mathrm{I}}^{\mathrm{b}}(\omega)
\end{aligned}
$$

where, using equation (3.11), the asymptotic behaviour of the 'band' contribution $\Sigma_{\mathrm{I}}^{\mathrm{b}}(\omega)$ is

$$
\Sigma_{\mathrm{I}}^{\mathrm{b}}(\omega) \stackrel{\omega \rightarrow 0}{\sim} \frac{3}{4} U^{2} q^{4} \Delta_{0}|\omega|^{r-2} .
$$

The low- $\omega$ behaviour of $\Sigma_{\mathrm{I}}(\omega)$ is thus integrably singular, and manifestly not that of a Fermi liquid or any natural generalization thereof: not surprisingly, since the underlying $U=0$ ground state for $r>1$ is a local moment one. The corresponding real part, $\Sigma_{\mathrm{R}}(\omega)$, 
follows from the Hilbert transform equation (2.6) using equation (5.10a), and has the leading low- $\omega$ behaviour

$$
\Sigma_{\mathrm{R}}(\omega) \stackrel{\omega \rightarrow 0}{\sim} \frac{U^{2} q^{3}}{4} P\left(\frac{1}{\omega}\right)
$$

(with corrections $\mathrm{O}\left(|\omega|^{r-2},|\omega|\right.$ ) arising from the transform of $\Sigma_{\mathrm{I}}^{\mathrm{b}}(\omega)$ ).

From equation (2.8) the low- $\omega$ behaviour of the impurity spectrum $D(\omega)$ thus follows directly as

$$
D(\omega) \stackrel{\omega \rightarrow 0}{\sim} \frac{\Sigma_{\mathrm{I}}^{\mathrm{b}}(\omega)}{\pi\left[\Sigma_{\mathrm{R}}(\omega)\right]^{2}}=\frac{12}{\pi} \frac{\Delta_{0}}{(U q)^{2}}|\omega|^{r} .
$$

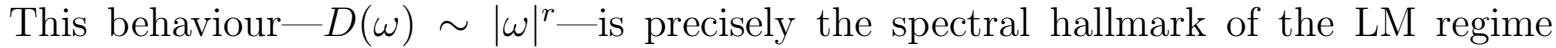
found in NRG calculations for $U>0$ [12]. It is of course in marked contrast to what obtains in the non-interacting limit, viz equation (3.11) $D_{0}^{\mathrm{b}}(\omega) \sim|\omega|^{r-2}$ (together with a pole contribution for $U=0$, which is eliminated entirely for $U>0$ ). Although the lowest frequency spectral asymptotics thus change character abruptly upon increasing $U$ from zero, it is however straightforward to show (using the asymptotic forms of $\Sigma_{\mathrm{R}} / \Sigma_{\mathrm{I}}^{\mathrm{b}}$ above) that for sufficiently small $U$ there exists a crossover scale $\omega_{0}=\frac{1}{2} U q^{2}$ such that for $(D \gg)|\omega| \gg \omega_{0}$ the behaviour of $D(\omega)$ is that of the non-interacting limit, viz $D(\omega) \sim|\omega|^{r-2}$; while for $|\omega| \ll \omega_{0}, D(\omega) \sim|\omega|^{r}$ as in equation (5.12).

Representative single-particle spectra for the LM state, obtained at the secondorder level, are illustrated in figures 3 and 4 for $r=1.5$. We consider first a 'weak hybridization' example which, for $r>1$, entails $\tilde{D}=\Delta_{0}^{\frac{1}{r-1}} D \ll 1$ (see equation (2.12)). For $\tilde{D}=1.5 \times 10^{-3}$, figure 3a shows the dimensionless $D^{\prime}(\tilde{\omega})=\Delta_{0}^{\frac{1}{1-r}} D(\omega)$ vs. $\tilde{\omega}=\omega / \Delta_{0}^{\frac{1}{1-r}}$ for three reduced interaction strengths $\tilde{U}=U / \Delta_{0}^{\frac{1}{1-r}}=2.5 \times 10^{-4}$, $5 \times 10^{-4}$ and $7.5 \times 10^{-4}$. The dominant visible feature of the spectra are the Hubbard satellites, which for all $\tilde{U}$ 's shown are centred to high accuracy on $\tilde{\omega}_{0}=\frac{1}{2} \tilde{U} q^{2}$ (with the poleweight $q \approx 0.95$ from equation (3.9) with $\delta=0$ ). This is expected physically: the weak hybridization regime is 'close' to the atomic limit, $\Delta_{0}=0=V_{i \boldsymbol{k}}$ (where $q=1$ ), which by a well known accident for the particle-hole symmetric case (see e.g. [1]) is captured exactly by second order PT, and where equation (5.11) with $q=1$ is exact for all $\omega$. The sharp Hubbard satellites of figure $3 \mathrm{a}$ thus correspond simply to weak resonant broadening, and only slight shifting, of the atomic limit poles occuring at $\omega= \pm \frac{1}{2} U$. The low- $\omega$ behaviour of the spectrum, equation (5.12), is not directly visible in figure $3 \mathrm{a}$, but is clear from the inset to figure $3 \mathrm{a}$ and in figure $3 \mathrm{~b}$. In the latter, for the same parameters as figure 3a, we show $\log \left[\left(\pi[\tilde{U} q]^{2} / 12\right) D^{\prime}(\tilde{\omega})\right]$ vs. $\log \tilde{\omega}$. That $D^{\prime}(\tilde{\omega}) \sim|\tilde{\omega}|^{r}$ as $\tilde{\omega} \rightarrow 0$ is evident; as too is the accuracy of equation $(5.12)$ in its entirety. The above mentioned crossover to $|\tilde{\omega}|^{r-2}$ behaviour for $|\tilde{\omega}| \gg \tilde{\omega}_{0}$ is also evident in figure $3 \mathrm{~b}$ for the lowest $\tilde{U}$ example.

The spectral features naturally evolve smoothly with increasing hybridization strength. For $r=1.5$ again, figure 4 shows $D^{\prime}(\tilde{\omega})$ vs. $\tilde{\omega}$ for a 'strong hybridization' example, $\tilde{D}=10$ (where $q \approx 0.2 \ll 1$ ), for $\tilde{U}=4,8$ and 20 . The low- $\tilde{\omega}$ behaviour, $D^{\prime}(\tilde{\omega}) \sim|\tilde{\omega}|^{r}$, is clearly seen in all cases. The Hubbard satellites are again centred on 
Magnetic impurities in gapless Fermi systems: perturbation theory
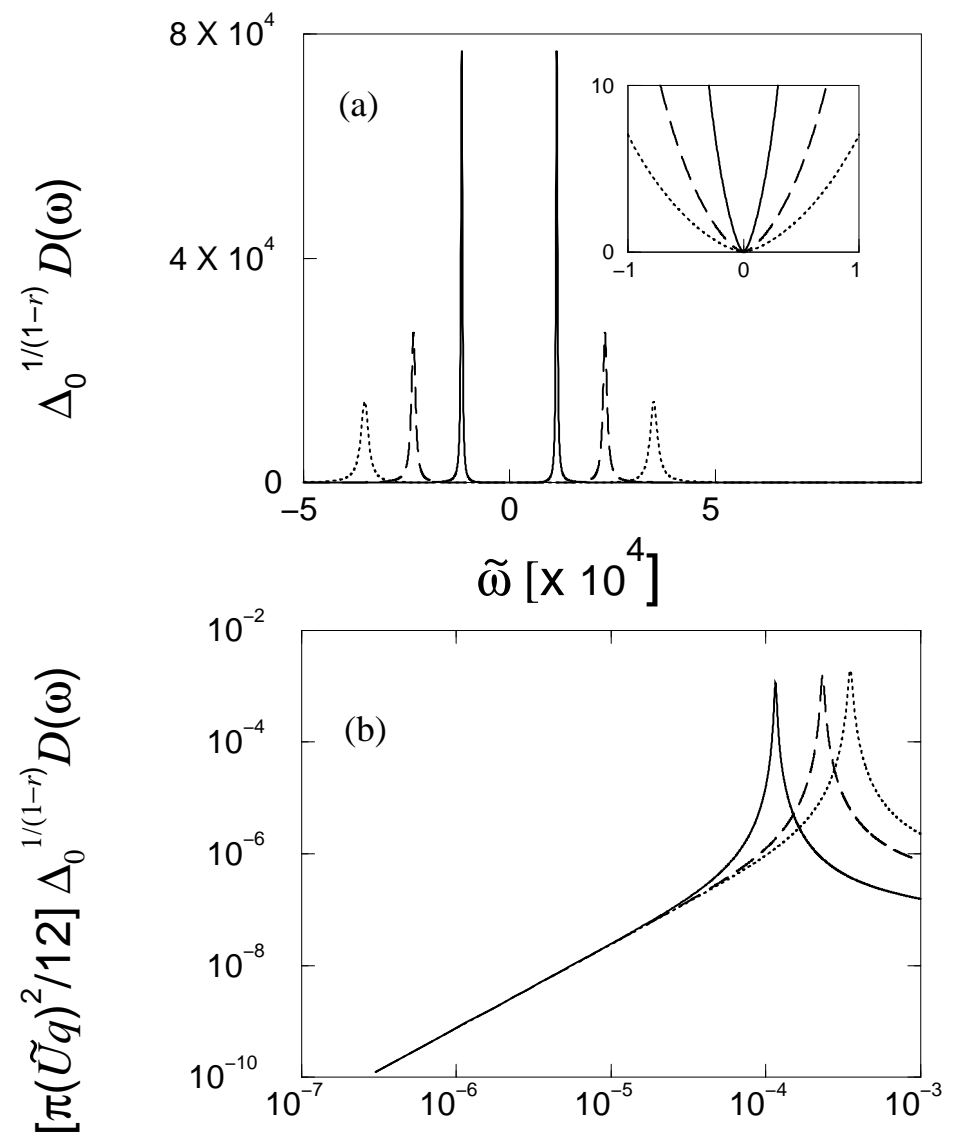

$\tilde{\omega}$

Figure 3. LM regime, $r=1.5$. (a) $D^{\prime}(\tilde{\omega})=\Delta_{0}^{\frac{1}{1-r}} D(\omega)$ versus $\tilde{\omega}=\omega / \Delta_{0}^{\frac{1}{1-r}}$ for reduced interaction strengths $\tilde{U}=2.5 \times 10^{-4}(-), 5 \times 10^{-4}(---)$ and $7.5 \times 10^{-4}$ $(\ldots \ldots)$; all for reduced bandwidth $\tilde{D}=1.5 \times 10^{-3}$. Inset: low- $\tilde{\omega}$ behaviour of $D^{\prime}(\tilde{\omega})$. (b) $\left[\pi(\tilde{U} q)^{2} / 12\right] D^{\prime}(\tilde{\omega})$ versus $\tilde{\omega}$ on a log-log scale for same parameters as (a).

$\tilde{\omega}_{0}=\frac{1}{2} \tilde{U} q^{2}$ for sufficiently low $\tilde{U}$ (e.g. for $\tilde{U}=4$ ); and in all cases, since $q \ll 1$, lie well below the scale of $\frac{1}{2} \tilde{U}$ characteristic of the atomic limit. It is also seen that the satellites become increasingly diffuse with increasing interaction strength, although whether this is a genuine feature is not clear since second order PT is by construction confined to weak coupling.

\section{2. $0 \leq r<\frac{1}{2}: S C$ state}

For $r<1$, $\operatorname{Im} 9 \Pi(\omega)$ is given by equation (5.9) and the $\omega \rightarrow 0$ behaviour of $D_{0}(\omega)\left(\equiv D_{0}^{\mathrm{b}}(\omega)\right)$ by equation (3.14). Hence from equation (5.5) the low frequency behaviour of $\Sigma_{\mathrm{I}}(\omega)$ is

$$
\Sigma_{\mathrm{I}}(\omega) \stackrel{\omega \rightarrow 0}{\sim} \alpha(U)|\omega|^{2-3 r}
$$




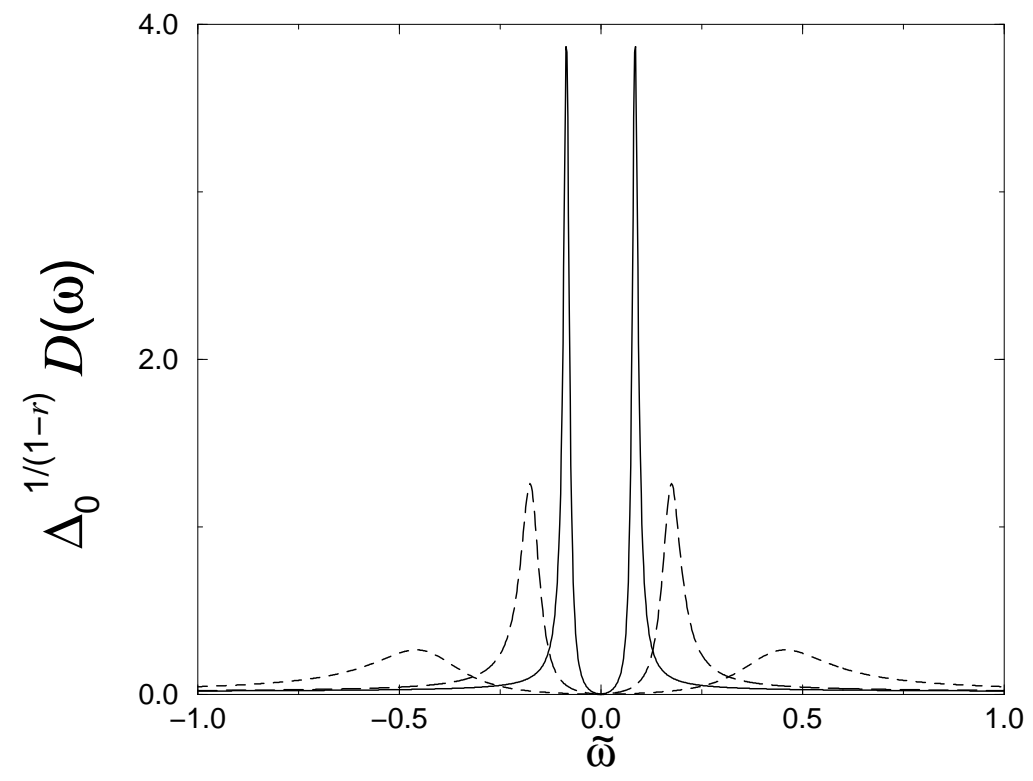

Figure 4. LM regime, $r=1.5$ and reduced bandwidth $\tilde{D}=10 . D^{\prime}(\tilde{\omega})$ versus $\tilde{\omega}$ for $\tilde{U}$ $=4(-), 8(---)$ and $20(---)$.

with

$$
\begin{aligned}
\alpha(U) & =U^{2} \frac{\cos ^{2}\left(\frac{\pi}{2} r\right)}{\Delta_{0}} C(r) \int_{0}^{1} \mathrm{~d} y y^{1-2 r}(1-y)^{-r} \\
& =U^{2} \frac{\cos ^{2}\left(\frac{\pi}{2} r\right)}{\Delta_{0}} C(r) \frac{\Gamma(2[1-r]) \Gamma(1-r)}{\Gamma(3[1-r])}
\end{aligned}
$$

and $C(r)$ given by equation (5.9). From the Hilbert transform equation (2.6), the corresponding behaviour of $\Sigma_{\mathrm{R}}(\omega)$ follows as

$$
\Sigma_{\mathrm{R}}(\omega) \stackrel{\omega \rightarrow 0}{\sim} \begin{cases}-\operatorname{sgn}(\omega) \gamma|\omega| & : 0 \leq r<\frac{1}{3} \\ -\operatorname{sgn}(\omega) \tan \left[\frac{\pi}{2}(2-3 r)\right] \alpha(U)|\omega|^{2-3 r} & : \quad r>\frac{1}{3}\end{cases}
$$

(with logarithmic behaviour $\sim|\omega| \ln |\omega|$ for $r=\frac{1}{3}$ ), where $\pi \gamma=\int_{-\infty}^{\infty} \mathrm{d} \omega \Sigma_{\mathrm{I}}(\omega) / \omega^{2}>0$.

The important point here is that for $r<\frac{1}{2}$, where $r<2-3 r, \Sigma_{\mathrm{I}}(\omega)$ and $\Sigma_{\mathrm{R}}(\omega)$ decay to zero as $\omega \rightarrow 0$ more rapidly than $\Delta_{\mathrm{I} / \mathrm{R}} \sim|\omega|^{r}$. The conditions established in section 4 for the SC state are thus satisfied at the level of second order perturbation theory, and the SC state is hence perturbatively continuable from the non-interacting limit. Interactions do not renormalize the asymptotic behaviour of $D(\omega)$ as $\omega \rightarrow 0$, and the generalized pinning condition equation (4.7) is thus satisfied. Note moreover that, as for the normal Anderson model $r=0, \Sigma_{\mathrm{I}}(\omega)$ vanishes at the Fermi level $\omega=0$.

Representative single-particle spectra for the SC phase are shown in figure 5 which, for $r=\frac{1}{4}$ and the wide-band limit $(D=\infty)$, shows $D^{\prime}(\tilde{\omega})$ vs. $\tilde{\omega}$ for $\tilde{U}=1,5$ and 7.5. As found in NRG studies [12], the gross features of the spectrum are dominated by the low- $\omega$ behaviour where $D(\omega) \sim|\omega|^{-r}$. And with increasing interaction strength $\tilde{U}$, 
the low frequency resonant continuum narrows and emergent Hubbard satellites become evident as weak shoulders in the spectrum.

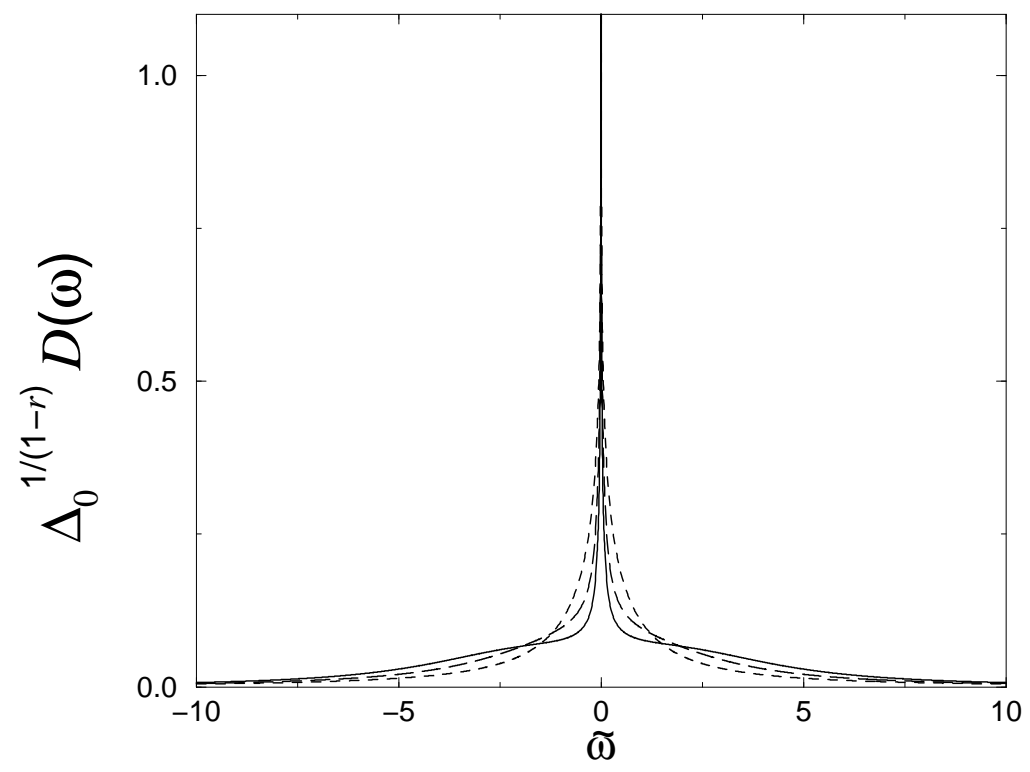

Figure 5. SC phase, $r=\frac{1}{4} . D^{\prime}(\omega)=\Delta_{0}^{\frac{1}{1-r}} D(\omega)$ versus $\tilde{\omega}$ for $\tilde{U}=1(---), 5(---)$ and $7.5(-)$.

A far more revealing exposé is seen in figure 6 where, for the same parameters as figure $5, \pi \Delta_{0}\left[1+\tan ^{2}\left(\frac{\pi}{2} r\right)\right] A(\omega)$ vs. $\tilde{\omega}$ is shown, with

$$
A(\omega)=|\omega|^{r} D(\omega) .
$$

Note first that the generalized pinning condition equation (4.7), which applies for any $r$ where a SC state obtains, is manifestly satisfied. The low- $\omega$ behaviour of $A(\omega)$ is cusp-like, and from the asymptotics given above it is readily shown that $A(\omega) \sim A(0)-\left[a_{1}|\omega|^{1-r}+a_{2}|\omega|^{2(1-2 r)}\right]$ where the coefficient $a_{1} \propto \tan (\pi r / 2)$. For $0<r<\frac{1}{3}$ the cusp is thus of form $\Delta A(\omega) \sim|\omega|^{1-r}$, while for $\frac{1}{3}<r<\frac{1}{2}$ it behaves as $\Delta A(\omega) \sim|\omega|^{2(1-2 r)}$; and for $r=0$, the parabolic behaviour $\Delta A(\omega) \sim \omega^{2}$ characteristic of 'normal' Fermi liquid behaviour is recovered. In fact, the behaviour of $A(\omega)$ shown in figure 5 for $r=0.25$ is strongly reminiscent of spectra characteristic of the normal Anderson model, $r=0$; the latter being shown in figure 6 for the same $\tilde{U}$ values. The parallels are obvious, in each case $A(\omega=0)$ being $\tilde{U}$-independent (pinned) and with Hubbard satellite peaks progressively evolving with increasing interaction strength. Most significantly, we see in either case the emergence with increasing $\tilde{U}$ of a low-energy scale, reflected in the half-width, $\omega_{K}$, of $A(\omega)$ which narrows progressively as $\tilde{U}$ is increased. For $r=0$ this is just the emergence of the ordinary Kondo scale, while for $0<r<\frac{1}{2}$ it is the generalization thereof known in a thermodynamic context for the soft-gap Kondo model itself $[6,10]$. 

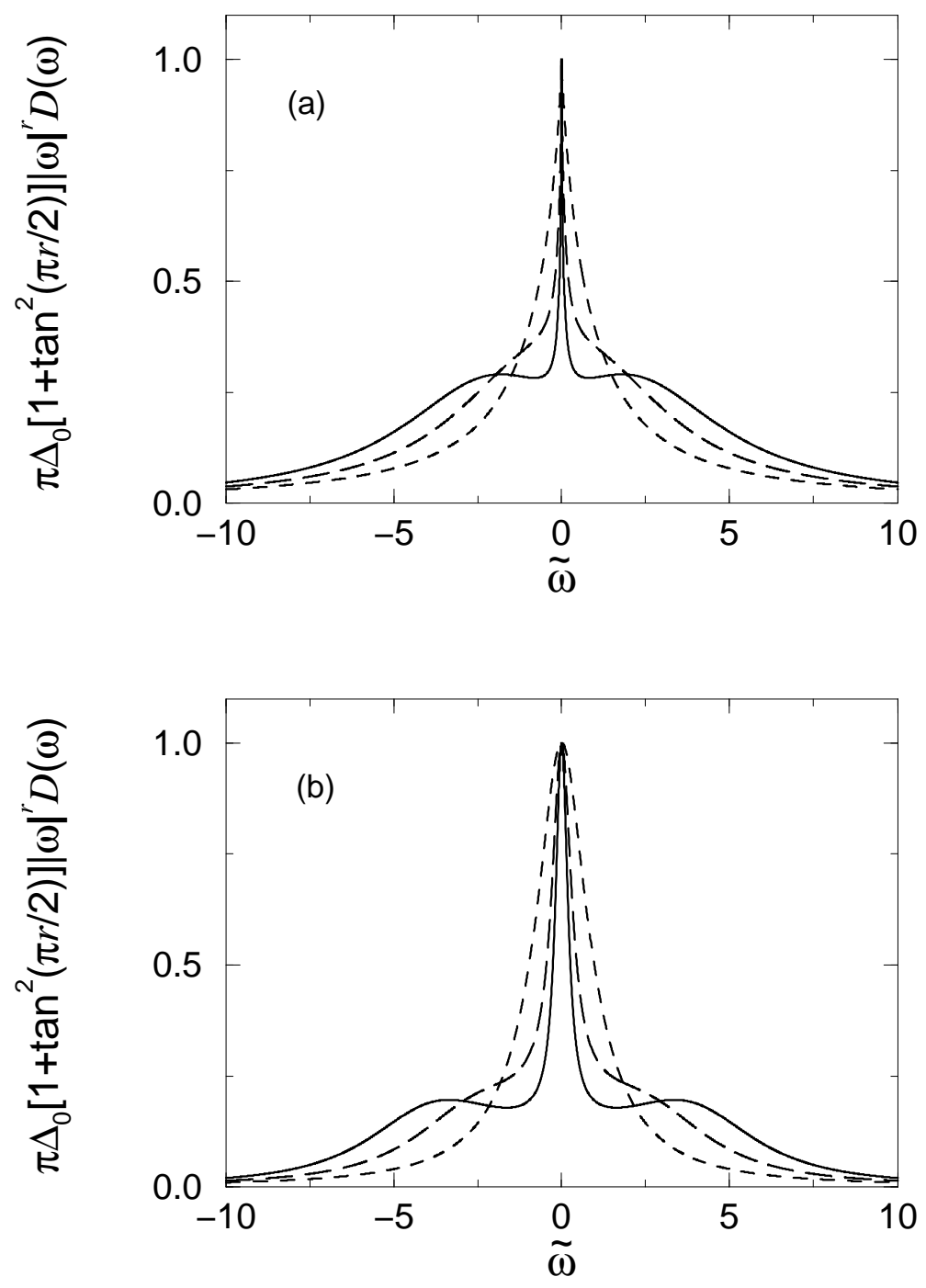

Figure 6. $\pi \Delta_{0}\left[1+\tan ^{2}\left(\frac{\pi}{2} r\right)\right]|\omega|^{r} D(\omega)$ versus $\tilde{\omega}$ for (a) SC phase, $r=\frac{1}{4}$ and (b) 'normal' $r=0$ Anderson model. For $\tilde{U}=1(---), 5(--)$ and 7.5 (- - in either case.

We shall not however pursue here the evolution of the Kondo scale with increasing $\tilde{U}$ since the above analysis, while showing that the SC state is perturbatively continuable from $\tilde{U}=0$ and enabling a description of the spectrum at low $\tilde{U}$, also points clearly to the limitations of a low-order perturbative treatment. For the normal Anderson model, we know that low-order perturbation theory is intrinsically incapable of describing strong coupling 'Kondo' asymptotics - $D(\omega)$ narrowing algebraically with increasing $\tilde{U}$ and failing thereby to yield the correct exponentially small Kondo scale $\omega_{K} \sim \exp \left(-\pi U / 8 \Delta_{0}\right)$ (see e.g. [1]). And the situation is even more acute for the SC phase with $r>0$ : here, from NRG studies of the gapless Anderson model [12,13], it is known that for $0<r<\frac{1}{2}$ there is a critical line $\tilde{U}_{C}(r)$ below which a SC state obtains, and above which the ground state is by contast a LM one. As $\tilde{U} \rightarrow \tilde{U}_{C}(r)-$, the low energy Kondo scale 
$\omega_{K}$ characteristic of the SC state must vanish, and this clearly cannot be captured by straight perturbation theory. To capture such behaviour, and hence in particular to describe the SC/LM phase boundary, an intrinsically non-perturbative approach is needed, and will be given in a subsequent paper [14].

\section{3. $\frac{1}{2}<r<1$}

Finally, we turn to the regime $\frac{1}{2}<r<1$. Here the low- $\omega$ asymptotics of the second order self-energy are again precisely those given in section 5.2, viz equations (5.13) and (5.14b). But in contrast to $0 \leq r<\frac{1}{2}, \Sigma_{\mathrm{I}}(\omega)$ and $\Sigma_{\mathrm{R}}(\omega)$ do not decay to zero as $\omega \rightarrow 0$ more rapidly than $\Delta_{\mathrm{I} / \mathrm{R}} \sim|\omega|^{r}$, whence the conditions established in section 4 for the SC state are not satisfied for $\frac{1}{2}<r<1$ : there is no pole contribution to $\Delta \rho(\omega)$, and the pinning condition equation (4.7) is not satisfied since the low- $\omega$ behaviour of the impurity spectrum is apparently dominated by $\Sigma_{\mathrm{I} / \mathrm{R}}$, viz $D(\omega) \sim\left[\Sigma_{\mathrm{I}}(\omega)\right]^{-1} \sim|\omega|^{3 r-2}$ diverging as $\omega \rightarrow 0$ for $\frac{1}{2}<r<\frac{2}{3}$, but less rapidly than the $|\omega|^{-r}$ behaviour characteristic of the SC state; and vanishing for $\frac{2}{3}<r<1$, but less rapidly than the $|\omega|^{r}$ behaviour symptomatic of the LM state. Superficially, therefore, one appears confronted by a state that is neither SC or LM in character.

There is, however, a plausible explanation: that straight perturbation theory in $U$ about the non-interacting limit is intrinsically inapplicable for $\frac{1}{2}<r<1$. We believe this to be the case, for we have shown in section 3 that for $U=0$ the ground state is SC for all $r<1$, and LM for $r>1$. By contrast, finite- $U$ NRG studies $[12,13]$ yield a LM ground state for $r>\frac{1}{2}$, with no indication of a transition between LM and $\mathrm{SC}$ states at any finite interaction strength. For $\frac{1}{2}<r<1$, therefore, the transition between SC and LM states occurs 'at' $U=0$ itself, and since the non-interacting and $U>0$ ground states are of different symmetry, one anticipates a breakdown of naive perturbation theory about the non-interacting limit. For $r<\frac{1}{2}$ or $r>1$ by contrast, the $U=0$ ground states are the same as those for $U>0$ (at least for sufficiently small $U$ in the case of $0<r<\frac{1}{2}$ ). Hence, as found in sections 5.1 and 5.2, one expects low order perturbation theory in $U$ to be applicable over some finite- $U$ interval; although we naturally anticipate such to become 'dangerous' as $r \rightarrow \frac{1}{2}-$ or $r \rightarrow 1+$.

\section{Conclusion}

A summary of the present work is aptly illustrated by figure 7 which, for the symmetric soft-gap Anderson model, shows schematically the phase boundaries between strong coupling (SC) and local moment (LM) phases in the $\tilde{U}=U / \Delta_{0}^{\frac{1}{1-r}}$ vs. $r$ plane. In the non-interacting limit, we have shown in section 3 that for $r<1$ the ground state is SC, while for $r>1$ it is LM. From finite- $U$ NRG studies [12,13] by contrast, it is known that the ground state is exclusively LM for $r>\frac{1}{2}$. In consequence, for $r>1$, second order perturbation theory in $U$ about the non-interacting limit as considered in section 5 appears able to capture at least the intial evolution of the LM state upon increasing 
$U$ from zero, yielding in particular the characteristic low-frequency spectral signature of the LM phase found in NRG studies [12], viz $D(\omega) \sim|\omega|^{r}$. For $\frac{1}{2}<r<1$ by contrast, the $U=0$ and $U>0$ ground states are fundamentally distinct, and we believe a finite-order perturbative treatment about the non-interacting limit to be simply inapplicable.

Perhaps the most subtle regime is $r<\frac{1}{2}$, encompassing as a particular case the 'normal' Anderson model, $r=0$. In section 4, general conditions were established for a SC state to be realized for $U>0$. Physically, these amount to interactions having no influence in renormalizing the lowest-frequency asymptotic behaviour of the impurity spectrum $D(\omega)$, whose behaviour as $\omega \rightarrow 0$ is that of the non-interacting limit, viz $D(\omega) \sim|\omega|^{-r}$ - producing the characteristic spectral signature of the SC state observed in NRG studies [12]. Significantly, one deduces in consequence a pinning condition (equation (4.7)) upon $A(\omega)=|\omega|^{r} D(\omega)$, whereby $A(\omega=0)$ at the Fermi level is pinned at its non-interacting value for all $U$ and $r$ where a SC state obtains, and which represents a natural generalization of the familiar pinning condition $D(\omega=0)=1 / \pi \Delta_{0} \forall U$ characteristic of the $r=0$ Anderson model. At the level of second order perturbation theory in $U$ (section 5.2), such a state of affairs is realized in practice for $r<\frac{1}{2}$, where the characteristic spectra $A(\omega)$ (figure 5) are strikingly reminiscent of their counterparts for $r=0$ (figure 6), with evolving Hubbard satellites upon increasing $U$ and the emergence of a low-frequency Kondo scale reflected in the progressively narrowing width of the generalized Abrikosov-Suhl resonance in $A(\omega)$.

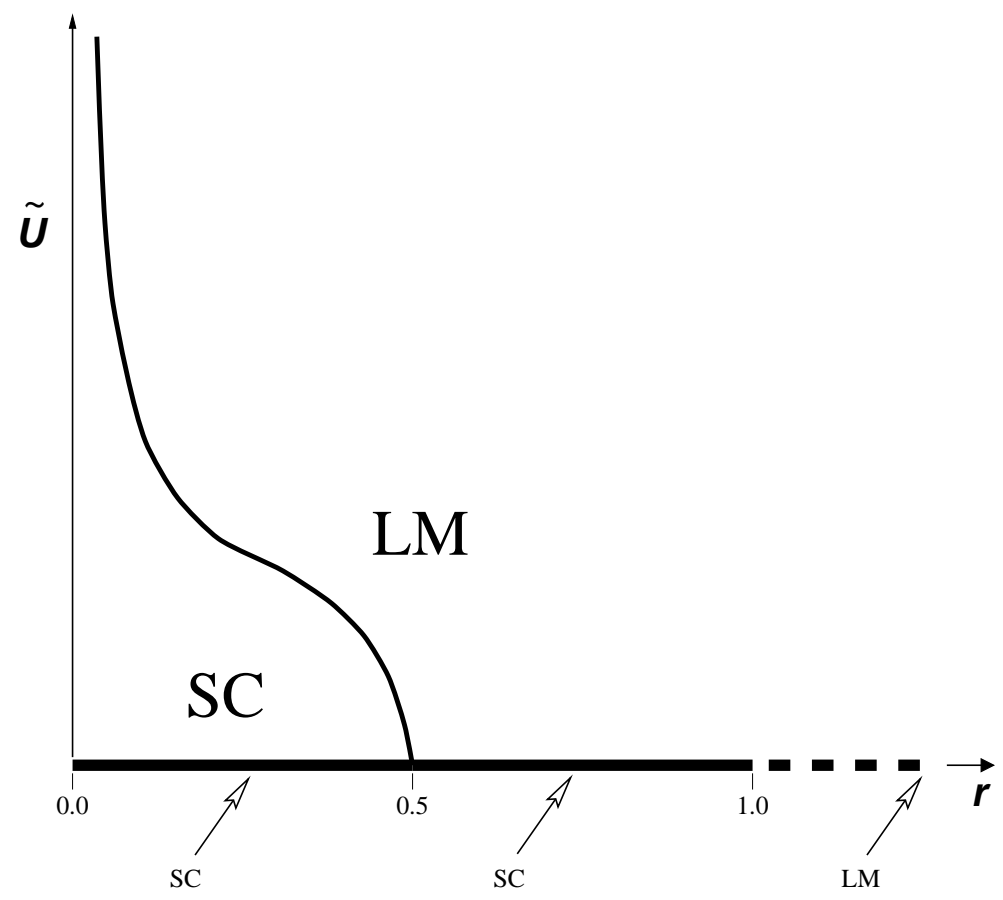

Figure 7. Schematic phase boundaries between SC and LM states in the $\tilde{U}=U / \Delta_{0}^{\frac{1}{1-r}}$ versus $r$ plane; details in text.

Figure 7 also shows clearly the limitations of finite order perturbation theory in 
the interaction strength. For $r<\frac{1}{2}$, NRG studies yield both SC and LM phases $[12,13]$, the critical line $\tilde{U}_{C}(r)$ diverging as $r \rightarrow 0$, reflecting the fact that the $r=0$ Anderson model is a Fermi liquid for all finite $U$; and vanishing as $r \rightarrow \frac{1}{2}$ (although whether it does so continuously as $r \rightarrow \frac{1}{2}-$ we regard as not wholly settled). It is in part this feature - the existence of a transition between a LM state and a SC (or generalized Fermi liquid) state - that renders the problem of generic interest. But low order perturbation theory, capable though it is of describing the initial evolution of the SC state upon increasing $U$ from zero, will naturally produce a SC state for all $U$ and cannot therefore delineate its boundary. To deal with this central issue, as well as to describe successfully the regime $\frac{1}{2}<r<1$, one requires an inherently non-perturbative theory that is capable of capturing both LM and generalized Fermi liquid phases, and hence the transition between them. For this, we believe a rather radical departure from conventional theoretical approaches is required, and will turn to one such in a subsequent paper [14].

\section{Acknowledgments}

We are grateful to R Bulla and T Pruschke for stimulating discussions. Financial support from the EPSRC and British Council is also gratefully acknowledged.

\section{References}

[1] Hewson A C 1993 The Kondo Problem to Heavy Fermions (Cambridge: Cambridge University Press)

[2] Volkov B A and Pankratov O A 1985 Pis. Zh. Eksp. Teor. Fiz. 42145 (1985 JETP Lett. 42 178)

[3] Sigrist M and Ueda K 1991 Rev. Mod. Phys. 63239

[4] Semenoff G W 1984 Phys. Rev. Lett. 532449

[5] Voit J 1995 Rep. Prog. Phys. 58977

[6] Withoff D and Fradkin E 1990 Phys. Rev. Lett. 641835

[7] Gonzalez-Buxton C and Ingersent K 1996 Phys. Rev. B. 5415614

[8] Borkovski L S and Hirschfeld P J 1992 Phys. Rev. B. 469274

[9] Cassanello C R and Fradkin E 1996 Phys. Rev. B. 5315079

[10] Chen K and Jayaprakash C 1995 J. Phys.: Condens. Matter 7 L491

[11] Ingersent K 1996 Phys. Rev. B. 5411936

[12] Bulla R, Pruschke Th and Hewson A C 1997 J. Phys.: Condens. Matter 910463

[13] Gonzalez-Buxton C and Ingersent K 1998 Phys. Rev. B. 5714254

[14] Logan D E and Glossop M T submitted for publication

[15] Nozières P 1974 J. Low Temp. Phys. 1731 